This is an original manuscript of an article published by ELSEVIER in Journal of NDT \& E International 107 (2019) on 31 July 2019, available online at: https://www.sciencedirect.com/science/article/pii/S0963869519300337?via\%3Dihub DOI: https://doi.org/10.1016/j.ndteint.2019.102152 


\title{
Comparison of Time and Frequency Domain Features' Immunity Against Lift-off in Pulse-Compression Eddy Current Imaging
}

\author{
Luigi Ferrigno ${ }^{1}$, Marco Laracca ${ }^{1}$, Hamed Malekmohammadi $^{2 *}$, Gui Yun Tian ${ }^{3}$, Marco Ricci $^{4}$ \\ ${ }^{1}$ Department of Electrical and Information Engineering, University of Cassino, 03043 Cassino (FR), Italy \\ $\{$ m.laracca,ferrigno\}@unicas.it \\ ${ }^{2}$ Department of Engineering, Polo Scientifico Didattico di Terni, University of Perugia, Strada di Pentima 4, \\ 05100 Terni, Italy \\ hamed.malekmohammadi@unipg.it \\ ${ }^{3}$ School of Electrical, Electronic and Computing Engineering, Newcastle University, \\ Merz Court, Newcastle upon Tyne, NE1 7RU, United Kingdom \\ g.y.tian@ncl.ac.uk \\ ${ }^{4}$ Department of Informatics, Modeling, Electronics and Systems Engineering, University of Calabria, \\ Via Pietro Bucci, 87036 Arcavacata, Rende CS, Italy \\ m.ricci@dimes.unical.it
}

\begin{abstract}
:
This paper reports a comparison between various imaging procedures based on time- and frequencydomain features applied to eddy current data collected on an Aluminium alloy benchmark sample containing a set of small notches with various depths. Historically, the pulsed eddy current method was introduced to improve the performance of eddy current testing in terms of increased penetration depth and lift-off invariance by exploiting time-domain features analysis precluded when using single- or multi-tone excitations. In this work, such analysis is instead accomplished by using a swept-frequency excitation signal along with an optimized pulse-compression procedure. This approach allows the user to deliver more energy to the system with respect to the pulsed approach, thus improving the resultant signal-to-noise-ratio (SNR) without losing the capability of time-domain feature extraction. The imaging procedure makes use of amplitude and phase features of both frequency- and time-domain data where time-phase is defined through the Hilbert transform of the pulse-compression output. Detection capability of various imaging strategies, namely A-, B- and C-scans, are compared in terms of inspection depth and lift-off robustness by using the SNR merit factor. It is shown that time-domain features outperform frequency-based ones in terms of SNR for the case of deeper defects and that phase features are robust against lift-off variations for both time and frequency domains. In addition, the analysis of time-amplitude images clearly evidences the presence of liftoff invariant points. To our knowledge, this is the first experimental evidence of the lift-off invariance points retrieved after applying pulse-compression in combination with coded excitation instead of using directly pulsed, multi-tone or single-tone sinusoidal signals. This not only confirms previous results achieved by the authors, but also demonstrates that pulse-compression eddy current can represent a valid solution to combine the advantages of pulsed and sinusoidal excitation strategies.
\end{abstract}

Keywords: Eddy Current Testing, Eddy current Imaging, Lift-Off Invariance (LOI), Pulse-Compression

\section{Introduction}

In the last decades, many research efforts have been devoted to increase the performances of Eddy Current Testing (ECT) mainly by means of Multi-Frequency (MF) or Pulsed Eddy-current (PEC) signals [18], or by exploiting electrical resonance phenomena [9]. By exciting the Sample Under Test (SUT) at several frequencies simultaneously or through time-multiplexing, the defect detection can be enhanced and at the same time useful information can be inferred about detects' depth and height through the analysis of the response at different frequencies. This is because the skin effect makes high frequencies less penetrating in the sample than low frequencies. Various strategies have been reported in literature to optimize the MF-ECT relying on both hardware and software solutions [10-14]. Proper probes capable of increasing the ECT 
sensitivity while lowering the effect of Lift-Off (LO) variation have been developed [15]; similar results have been also reached by using coded excitations, tailored signal and image processing procedures [16-17].

On the other hand, PEC uses short excitation signals that excite a continuous band of frequencies covering all the range of interest for the specific sample [1,4,7]. Time-analysis of PEC signals has proved to be extremely powerful in association with feature extraction procedures for the sake of both defect detection and characterization [18-19] and in addition PEC signals exhibit characteristic Lift-Off Invariance (LOI) points that can be useful to reduce LO noise [20-23]. Starting from PEC data, frequency analysis can be implemented by exploiting the Fourier analysis. Therefore, PEC is significantly richer in information than MF-ECT, but it is generally characterized by a lower Signal to Noise Ratio (SNR) values since MF signals are more energetic. This amount of information is usually not completely exploited since most of the PEC applications deals only with time-domain analysis. As a matter of fact, few works in literature compares the effectiveness of the two approaches and the possibility to gain advantages by the contextual usage of both [24-26].

In this framework, the goal of the present paper is to quantitatively compare the performance of imaging procedures proposed for frequency- and time-domain features in terms of SNR, and qualitatively in terms of defect detection capability of each feature. For the fair comparison in both time- and frequency-domain, an imaging procedure is implemented relying on the theory of Eddy Current (EC) diffusion. In addition, as a peculiar result of the present paper, instead of starting from PEC data, the time- and frequency- domain analysis is implemented by using a Swept Frequency (SF) "Chirp" excitation in combination with PulseCompression (PuC), henceforth referred as PuC-ECT. This approach allows the typical PEC output signals to be retrieved after the application of the PuC while assuring contextually the typically high SNR values of MF analysis, thus combining the positive characteristics of both techniques. A further goal is to evaluate the robustness of the various features proposed in this paper, against LO noise and verifying if LOI points are retrieved on the impulse responses estimated trough $\mathrm{PuC}$.

The paper is organized as follows: in Section II the basic theory of eddy current is summarized highlighting its diffusive nature. Starting from this, in Section III the imaging procedures for both time and frequency domains are illustrated. Section IV elaborates on the combination of swept-frequency "chirp" signals and PuC theory to implement the proposed PuC-ECT technique. The experimental setup and the data collection procedure are described in Section V while in Section VI the experimental results are illustrated. Conclusions and perspectives are drawn in Section VII.

\section{Theoretical background on Eddy Current diffusion theory}

The generation and the detection of EC inside a conductive material depend on the physical and geometrical properties of the SUT and are governed by the Maxwell's equations. In the application of the Maxwell's equations three different frequency regimes can be considered: static, quasi-static and timevarying. In the ECT application the second case is usually considered, which is the (magneto) quasi-static approximation in a conductive non-magnetic medium [27]. In a good conductor for which $\sigma / \omega \varepsilon \gg 1$, the displacement current $\bar{D}=j \omega \varepsilon \bar{E}$ is dependent on the frequency and its value is negligible for low frequencies, hence considered null. For more details on the basic theory of electromagnetism, the reader is referred to the existing literature, see for instance [28].

In the quasistatic approximation, the ECT physics is described as a diffusive phenomenon and indeed one can derive from the Maxwell's equations the following magnetic diffusion equations in 3D

$$
\begin{aligned}
& \partial \bar{J} / \partial t=1 / \mu \sigma \nabla^{2} \bar{J} \\
& \partial \bar{B} / \partial t=1 / \mu \sigma \nabla^{2} \bar{B} \\
& \partial \bar{E} / \partial t=1 / \mu \sigma \nabla^{2} \bar{E}
\end{aligned}
$$

where the term $\alpha_{m}=1 / \mu \sigma$ is the 'Magnetic Diffusivity' and indicates how fast the electric and magnetic fields can penetrate i.e. diffuse inside the conductive sample and reach the steady-state after applying an excitation [29-30].

Let assume for simplicity, a 1D ideal problem in which an electromagnetic plane-wave propagates along $z$ direction, coming from negative $z$ values and impinging perpendicular to the inspection surface of a semi- 
infinite SUT placed at $z=0$. The steady-state solution to Eq. 1(a) for the conduction current density inside the SUT, i.e. $z \geq 0$, can be written in phasor notation as:

$$
\bar{J}(z, t)=\bar{J}(0, t) e^{-\frac{z}{\delta}} e^{-j\left(\frac{z}{\delta}-\omega t\right)}=\bar{J}(0, t) e^{-\frac{z}{\delta}} e^{j \omega\left(t-\frac{z}{\omega \delta}\right)}
$$

where $\delta(f)$ is the 'Skin Depth' and is defined as:

$$
\delta(f)=\frac{1}{\sqrt{\pi \mu \sigma f}}=\sqrt{\frac{2}{\omega \mu \sigma}}
$$

Considering a sinusoidal excitation, in Eq. 2 the term $\frac{1}{\delta}$ is the angular wavenumber $k$ and therefore, the known quantity $v_{p}=\frac{2 \pi f}{k}$ or in this case the 'Phase Velocity' of the eddy current diffusion can be written as:

$$
v_{E C}(f)=2 \pi f \delta(f)=2 \sqrt{\pi f \alpha_{m}}
$$

Although the model considered is very simple, the solution to this problem is useful to describe the EC phenomenon and indeed the skin depth defined in Eq. 3 is at the basis of the ECT technique. In addition, by considering Eq. 3 and Eq. 4 together, which is rarely exploited, it is possible to define similar imaging procedures for both time- and frequency- domains and hence compare, fuse and evaluate both approaches. To provide a better insight into the proposed imaging procedures, more details about the physics behind the previous equations is necessary.

Eq. 2 does not represent a propagating wave but instead it describes an evanescent wave whose magnitude decays exponentially with depth while its phase angle varies linearly with depth but non-linearly with the frequency. Precisely, the exponential decay of the amplitude is regulated by the well-known Skin Depth $\delta(f)$ parameter and the phase lag is determined by the phase velocity $v_{E C}(f)$. Therefore, each frequency $f$ is associated with a characteristic inspection range (depth) $\delta(f)$ and a characteristic velocity of diffusion $v_{E C}(f)$. Signals with higher frequencies penetrate less inside the SUT and diffuse faster, while lower frequency ones diffuse in larger depths but slower. If a broadband excitation is used instead of a single frequency, the diffusion is also associated to a dispersive process that modifies the excitation waveform depending on the depth inside the sample.

At the same, it is possible to associate to a given depth $d$ from the surface inside the sample two characteristic frequency and time values, denoted by $f^{*}(d)$ and $t^{*}(d)$ respectively and expressed by the following relations:

$$
f^{*}(d)=\left(\pi \mu \sigma d^{2}\right)^{-1} \Rightarrow \delta\left(f^{*}(d)\right)=d ; \quad t^{*}(d)=(2 * d) / v_{E C}\left(f^{*}(d)\right)=\left(\mu \sigma d^{2}\right)
$$

The function $f^{*}(d)$ indicates the frequency value for which the skin depth $\delta\left(f^{*}(d)\right)$ is equal to $d$. Thus, $f^{*}(d)$ is the optimal frequency to detect inner defects placed at distance $d$ from the inspection surface. The function $t^{*}(d)$ gives the diffusion time needed for an evanescent wave of frequency $f^{*}(d)$ to reach the distance $d$ from the surface and come back when "reflected" by an inhomogeneity such a void, crack and generic defects. These two quantities relate nonlinearly depth with frequency and time by considering the diffusion law and starting from these relations it is possible to define a $3 \mathrm{D}$ imaging procedure for both frequency- and time-domain features.

It is worth to note that in real cases, the expressions in Eq. 5 must be corrected to consider the shape of the exciting coil, SUT's geometrical characteristic, and the spectrum of the excitation signal. However, these basic relations are still useful to design the excitation signal and to define the signal and image processing algorithms, as will be illustrated in the next section. 

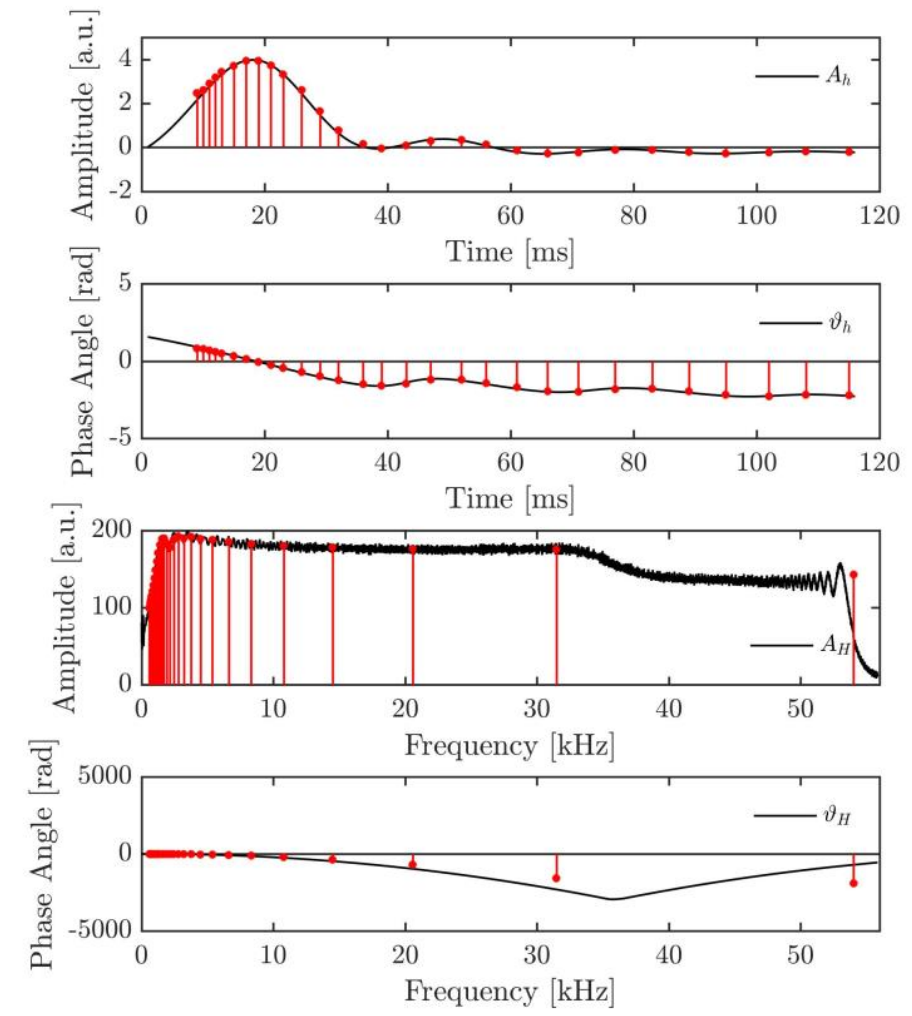

Fig.1. Example of the time and frequency features extraction from the output signal based on linear sampling in the inspection range $\zeta$
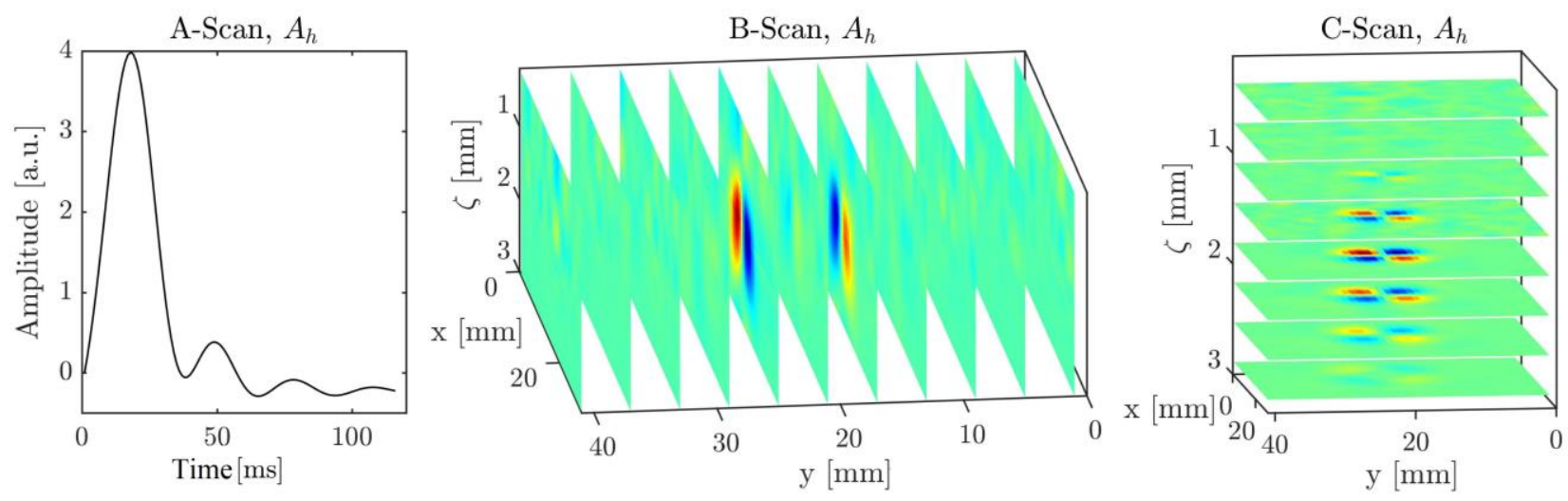

Fig. 2. Example of the imaging procedure. From left to Right: Impulse response of a single scan point presented as Ascan, B-scan and C-Scan acquired based on the set of A-Scan data for the same scan area

\section{Imaging procedure for time- and frequency-domain}

To develop the imaging procedures, following ideal procedure was considered: a short pulse excites the system in a frequency range $\left[f_{1}, f_{2}>f_{1}\right]$ covering the inspection range $\zeta$ of interest, i.e. $\zeta \in\left[\delta\left(f_{1}\right), \delta\left(f_{2}\right)\right]$ and with a constant excitation power spectrum.

Data are collected on a regular $x-y$ grid of $N_{x} \times N_{y}$ points over the SUT's inspection surface and for each point the time response $\mathrm{y}(t, x, y)$ is collected. If $\left[f_{1}, f_{2}\right]$ interval is large enough, $\mathrm{y}(t, x, y)$ can be considered as a good approximation of the impulse response $h(t, x, y)$ of the SUT, collected at any $x-y$ measurement point. Here and henceforth this condition is assumed to be satisfied and $h(t, x, y)$ will be used to indicate a single measurement output.

Starting from the measurement of $h(t, x, y)$, by applying the Fourier transform, $H(f, x, y)=$ $\mathcal{F}\{\mathrm{h}(t, x, y)\}$, the amplitude $A_{H}(f, x, y)=|H(f, x, y)|$ and the phase $\vartheta_{H}(f, x, y)=\operatorname{Arg}\{\mathrm{H}(f, x, y)\}$ of the spectrum can be obtained. In the time domain, to obtain a full analogy between the two approaches, the time-phase concept in ECT is introduced by exploiting the Hilbert Transform as proposed in thermography 
NDT by Mandelis and co-workers and then applied by various authors [31-32]. Firstly, the analytic signal $\Gamma_{h}(t, x, y)=h(t, x, y)+j \mathcal{H}\{h(t, x, y)\}$ is calculated, where $\mathcal{H}\{\cdot\}$ is the Hilbert transform, then the timeamplitude $A_{h}(t, x, y)=h(t, x, y)$ and time-phase $\vartheta_{h}(t, x, y)=\operatorname{Arg}\left\{\Gamma_{h}(t, x, y)\right\}$ are extracted. Hence, for each measurement point, four signals as obtained, one for each of the four features.

Starting from these four signals, the imaging procedures are implemented by sampling the signals to provide linearly spaced samples in inspection depth instead of time and frequency. According to the theory of EC diffusion introduced in Section II, considering the desired inspection depth $\zeta$ being linearly subdivided to $N_{\zeta}$ intervals, such that: $\vec{\zeta}=\left[\zeta_{1}, \zeta_{1}+d \zeta, \zeta_{1}+2 \cdot d \zeta, \cdots, \zeta_{1}+N_{\zeta} \cdot d \zeta\right]$, where $\mathrm{d} \zeta$ is the desired depth resolution, i.e. $d \zeta=\zeta / N_{\zeta}$, both time and the frequency axes can be subdivided in $N_{\zeta}$ intervals as well. The resulting time and frequency axes are defined by the two non-linearly spaced vectors: $\vec{f}=f^{*}(\vec{\zeta})=$ $\pi \mu \sigma\left(\vec{\zeta}^{2}\right)^{-1}$ and $\vec{t}=t^{*}(\vec{\zeta})=\mu \sigma\left(\vec{\zeta}^{2}\right)$ and the average values of $A_{H}, \vartheta_{H}, A_{h}, \vartheta_{h}$ are calculated for each depth interval. At the end of the procedure four vectors $\overrightarrow{A_{H}}[m], \overrightarrow{\vartheta_{H}}[m], \overrightarrow{A_{h}}[m], \overrightarrow{\vartheta_{h}}[m]$, with $m \in\left[1, N_{\zeta}\right]$, are obtained that are used in the image formation step. Figure 1 summarizes the sampling procedure described above.

To use the same terminology adopted in ultrasonic NDT and henceforth for clarity, these four vectors will be considered as A-scan data sets. Once all four $N_{x} \times N_{y}$ A-scans are retrieved, they can be combined to generate B-scans along $x-\zeta$ or $y-\zeta$ planes at a given $x_{k}$ or $y_{l}$ value respectively and C-scans for each $\zeta_{m}$ value, i.e. depth, as depicted in Figure 2 for the $\mathrm{A}_{h}$ feature.

The aim of this paper is to quantitatively and qualitatively compare the B- and C-scan images obtained with the four features, i.e. $A_{H}, \vartheta_{H}, A_{h}, \vartheta_{h}$, in term of defect detectability and image SNR in order to compare and evaluate the efficiency of time-domain analysis with respect to the frequency-domain analysis. To accomplish this goal, a crucial point is the measurement of the set of responses $h\left(t, x_{k}, y_{l}\right), k \in\left[1, N_{x}\right], l \in$ $\left[1, N_{y}\right]$ by using swept-frequency PuC-ECT instead of PEC, as will be explained in the next section.

\section{Swept-Frequency excitation and Pulse compression-ECT}

The diffusive nature of EC hampers the detection of deep defects due to the exponential decrease of the eddy current density within the SUT's thickness and the associated dispersion phenomenon, see Eq. 2. As a result, the SNR of ECT signals due to deep defects is usually very low affecting the quality of A-, B- and Cscans introduced in Section III. To increase the SNR of signals and related images, and hence improving contextually the defect detection capability, PuC can be used in ECT in combination with both sweptfrequency or pseudo-noise signals $[14,26]$ instead of standard PEC.

$\mathrm{PuC}$ is a technique developed for estimating the impulse response $h(t)$ of a Linear Time Invariant (LTI) system in noisy environments and more general, when high SNR values must be achieved. If a nonferromagnetic SUT is considered and non-linear phenomena in the sensors are avoided, an ECT measurement setup can be modelled as an LTI. Hence, PuC-ECT can be successfully applied. Here only the useful key aspects for the present procedure will be summarized. For a detailed theory of PuC, the reader is referred to [33,34].

Suppose that in a PEC scheme the SUT is excited by a short pulse $s(t)$ that can be considered a good approximation of the Dirac's delta function: $s(t) \sim \delta(t)$. Since realization of an ideal Dirac's delta is not practically possible, this approximation is acceptable if the bandwidth of $s(t)$ covers all the range of characteristic frequencies of the defects' signals. For each $\left(x_{k}, y_{l}\right)$ measurement point the output will be $y\left(t, x_{k}, y_{l}\right)=s(t) * h\left(t, x_{k}, y_{l}\right) \sim h\left(t, x_{k}, y_{l}\right)$, where $h\left(t, x_{k}, y_{l}\right)$, as previously mentioned, is the SUT's impulse response at any measurement point and $*$ is the convolution operator. In PuC-ECT, instead of exciting the SUT with $s(t)$, the excitation is provided through a coded signal $x(t)$ covering the same frequency range of interest as $s(t)$ but having an arbitrary duration. The new output signal $y_{P u C}\left(t, x_{k}, y_{l}\right)$ is described by the convolution as follows: $y_{\text {PuC }}\left(t, x_{k}, y_{l}\right)=x(t) * h\left(t, x_{k}, y_{l}\right)$.

If exist another signal $\psi(t)$ such that $x(t) * \psi(t) \sim s(t), h\left(t, x_{k}, y_{l}\right)$ can be then retrieved by filtering $y_{P u C}\left(t, x_{k}, y_{l}\right)$ with $\psi(t)$. Indeed, we obtain:

$$
h_{P u C}\left(t, x_{k}, y_{l}\right)=\psi(t) * y_{P u C}\left(t, x_{k}, y_{l}\right)=\underbrace{\psi(t) * x(t)}_{\sim s(t)} * h\left(t, x_{k}, y_{l}\right) \sim s(t) * h\left(t, x_{k}, y_{l}\right) \sim h\left(t, x_{k}, y_{l}\right)
$$

$\psi(t)$ is called 'matched filter' and in most of the PuC schemes $\psi(t)$ is nothing but the time-reversed input signal amplitude modulated by a proper weighting/window function $w(t): \psi(t)=w(t) x(-t)$ [33]. The 


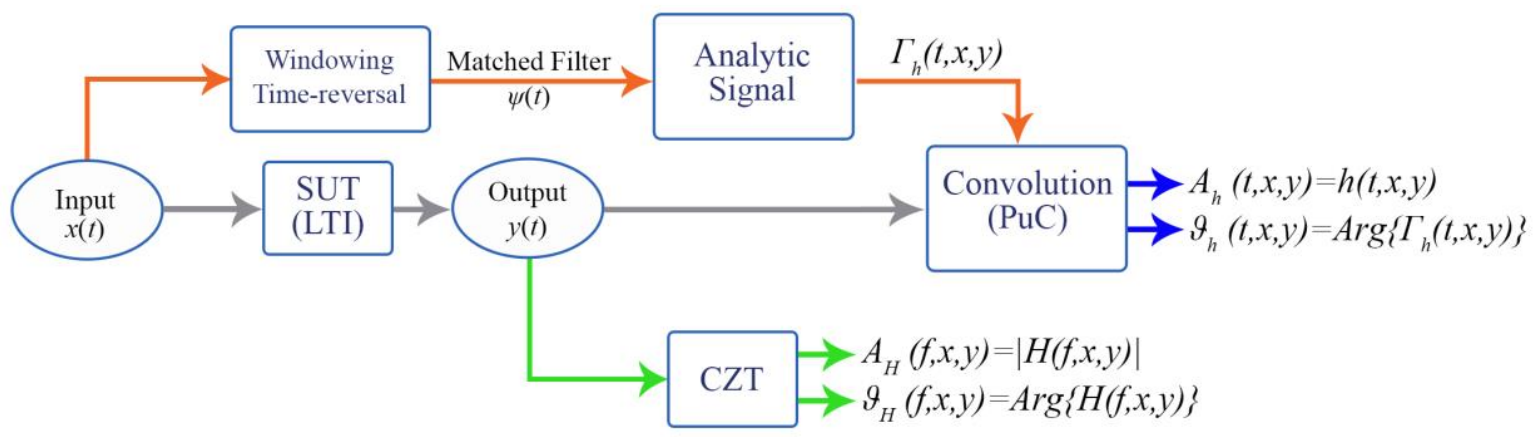

Fig. 3. Schematic representation of time analysis and PuC scheme providing as output the analytic signal of the estimated impulse response

choice of $w(t)$ is made to optimize the trade-off between $S N R$ gain and reconstruction fidelity for the specific application. In this paper, in order to retrieve a good estimate of $h\left(t, x_{k}, y_{l}\right)$ the $w(t)$ was selected based on reactance transformation [34] that allows the maximal reduction in sidelobes of impulse response after PuC.

Practically, the main advantage of the using of $\mathrm{PuC}$ is that when $x(t)$ is a proper coded signal, its duration $T$ is almost independent of its bandwidth $B$; this allows delivering more energy to the system, hence increasing the SNR. In recent years, PuC has been applied in ECT by some of the authors using various excitation signals. In this paper the linear swept-frequency "chirp" signal is employed, although the use of properly designed non-linear chirp signals could be beneficial to further improve the SNR for deeper defects [34].

A generic "chirp" signal is a sinusoidal signal characterized by a nonlinear accumulated phase function $\Phi(t): x(t)=A \sin (\Phi(t))$. An instantaneous frequency is defined as $f_{i s t}(t)=\frac{1}{2 \pi} \frac{d \Phi(t)}{d t}$ and starting from an arbitrary continuous trajectory of $f_{\text {ist }}(t)$ in the time interval of 0 to $T$, the related chirp signal could be obtained by calculating $\Phi(t)$ by integration. When $f_{i s t}(t)$ is a linear function of time, the chirp is called "linear". In this latter case, $f_{\text {ist }}(t)$ is usually expressed by $f_{\text {ist }}(t)=f_{1}+\left(f_{2}-f_{1}\right)^{t} / T$, where $f_{1}$ and $f_{2}$ are the start and stop frequencies respectively. The resulting phase is given by: $\Phi(t)=2 \pi\left(f_{1} \cdot t+\frac{B}{2 T} \cdot t^{2}\right)$ where $B=\left(f_{2}-f_{1}\right)$. The chirp bandwidth is equal to $|B|$ and if $f_{2}>f_{1}$ the chirp is called "UP", otherwise "DOWN". Linear chirp is undoubtedly the most used waveform in PuC schemes such as RADAR, ultrasonic inspections, acoustics, etc. Henceforth we consider only linear UP chirp signals for which $|B|=B$.

The instantaneous frequency is also strictly related to the Power Spectral Density (PSD) of the resulting chirp. For a linear chirp, if the time-bandwidth product is large enough T $\cdot \mathrm{B} \gg 1$, the PSD is almost flat and confined within the interval $\left[f_{1}, f_{2}\right]$.

Starting from a linear chirp excitation signal, for each measurement point the four features are extracted according to the block diagram reported in Figure 3: the Fourier analysis is directly applied to the chirp output signal $y_{\text {PuC }}\left(t, x_{k}, y_{l}\right)$ for retrieving $A_{H}(f, x, y)$ and $\vartheta_{H}(f, x, y)$. This is because $x(t)$ covers uniformly the frequency range of interest. The time-domain signals, $A_{h}(f, x, y)$ and $\vartheta_{h}(f, x, y)$ are retrieved after the application of the PuC. In particular, to contextually calculate the PuC output and its Hilbert transform, $y_{P u C}\left(t, x_{k}, y_{l}\right)$ is filtered by the analytic signal of the matched filter $\psi(t)$, i.e. $\Gamma_{\psi}(t)$, to retrieve the analytic signal of the estimated impulse response $\Gamma_{h}(t)$. 


\section{V. $\quad$ Benchmark sample and measurement setup}

Measurement scans and the subsequent imaging procedure were executed on a specimen with known artificial defects. The specimen was a plate made of 2024-T3 aluminium alloy with an electric conductivity equal to $18.8 \mathrm{MS} / \mathrm{m}$, a magnetic permeability of $1.26 \mathrm{H} / \mathrm{m}$, and a thickness of $2 \mathrm{~mm}$.

All the defects were small notches having a length of $3 \mathrm{~mm}$ and width of $0.1 \mathrm{~mm}$, with varying depths from the inspection surface. Starting from $\mathrm{D}_{1}$ to $\mathrm{D}_{8}$ their depth varied respectively from $1.6 \mathrm{~mm}$ to $0.2 \mathrm{~mm}$ with a step of $0.2 \mathrm{~mm}$. $\mathrm{D}_{9}$ is a through-hole notch. For each defect except $\mathrm{D}_{9}$, a set of $\left[\mathrm{N}_{x}=41 \times \mathrm{N}_{y}=31\right]$ measurements were collected over a regular grid of $1 \mathrm{~mm} \times 1 \mathrm{~mm}$. Figure 4 depicts a sketch of the sample and one of the typical scans realized over a defect.

The ECT probe used was the same described in $[12,14]$ consisting of a rectangular excitation coil, a GMR sensor, a permanent magnet and a signal conditioning system. The excitation coil was realized with 288 turns and had dimensions of $22 \mathrm{~mm} \times 10 \mathrm{~mm} \times 1.6 \mathrm{~mm}$. During the scans, its main axis was oriented parallel to the sample surface and aligned along the notches' direction. The GMR sensor (NVE- AAH002-02) measured the component of the B field parallel to the surface and perpendicular to the coil axis, i.e. perpendicular to the notches' direction. A permanent magnet biased the field measured by the GMR to $30 \%$ of the saturating field value. This allowed working in the range of linear sensitivity of the sensor.

Each scan was realized by keeping constant the LO value of the sensor with respect the sample. In addition, for the defects from $\mathrm{D}_{5}$ to $\mathrm{D}_{8}$ scans at four different $\mathrm{LO}$ values $(0 \mathrm{~mm}, 1 \mathrm{~mm}, 2 \mathrm{~mm}, 3 \mathrm{~mm})$ were collected to test the imaging procedure in the case of $\mathrm{LO}$ variation.

The block diagram of the whole measurement setup is reported in Figure 5. A virtual instrument (VI) realized with LabVIEW ${ }^{\mathrm{TM}}$ generated the digital signal that was sent to an Arbitrary Waveform Generator (AWG- Agilent ${ }^{\mathrm{TM}} 33220 \mathrm{~A}$ ) implementing the digital to analog (D/A) conversion of the selected excitation waveform. The AWG output drove a power amplifier (20-20 Kepko ${ }^{\mathrm{TM}}$ Bipolar Power Operational Amplifier) that furnished the power to supply the EC-Probe with the excitation signal $x(t)$. A digital multimeter (Agilent ${ }^{\mathrm{TM}}$ 34401A) allowed the optional measurement of the excitation current for checking the correspondence with the wanted amplitude. The ECT probe was moved on the SUT by means of an $x-y$ translation system driven by the VI. The output voltage of the GMR sensor $y_{G M R}(t)$ was conditioned and processed to retrieve the measurement information: $y_{G M R}(t)$ was sent to a signal conditioning unit consisting of a cascade of a Analog Devices ${ }^{\mathrm{TM}}$ AD620 instrumentation amplifier with unitary gain and a Stanford Research System SR 560 bandpass filter. The conditioned signal was digitized by means of a National Instruments ${ }^{\mathrm{TM}}$ analog-to-digital (A/D) conversion board. Finally, the output digital signal $y_{P u C}[n]$ was sent to the VI to be stored and further processed.

The signal used to excite the coil was a linear chirp with a wide bandwidth starting from $f_{l}=540 \mathrm{~Hz}$ to

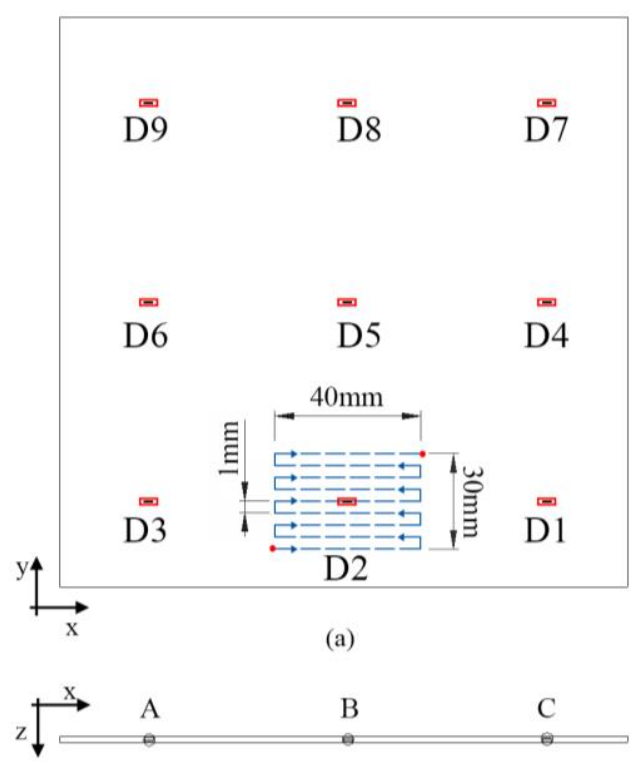

(b)

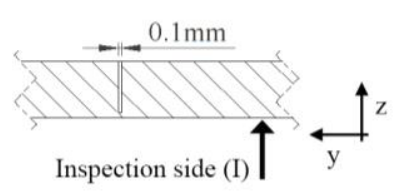

(e)

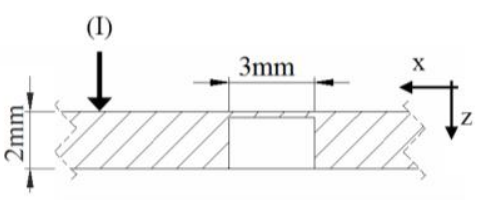

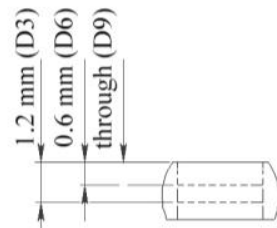

A

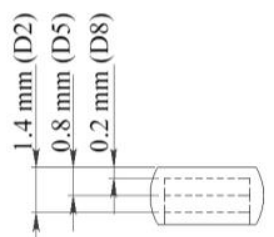

B

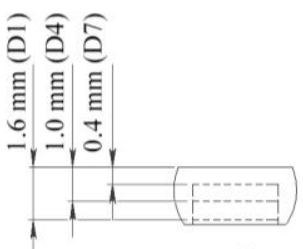

$\mathrm{C}$ (c)

Fig. 4. (a) Top view of the sample with 9 defects and a sample scan grid path around defect D2 (b) Side view of the sample (c) Detailed views of A, B and C (d) Side view of the defect D8 (e) Front view of defect D8 
$f_{2}=54 \mathrm{kHz}$ with a duration of $40 \mathrm{~ms}$. According to Section IV, the time-bandwidth product was quite large, $T B \approx 2000$, ensuring a high SNR value as well as a flat spectrum over all the bandwidth.

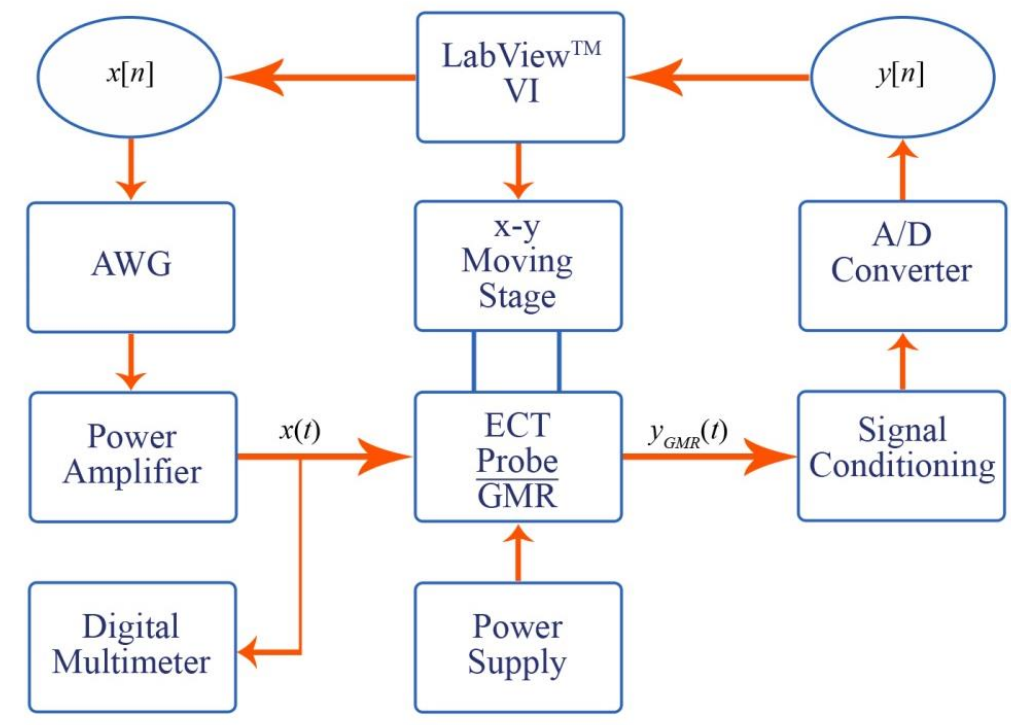

Fig. 5. Block diagram of the measurement setup
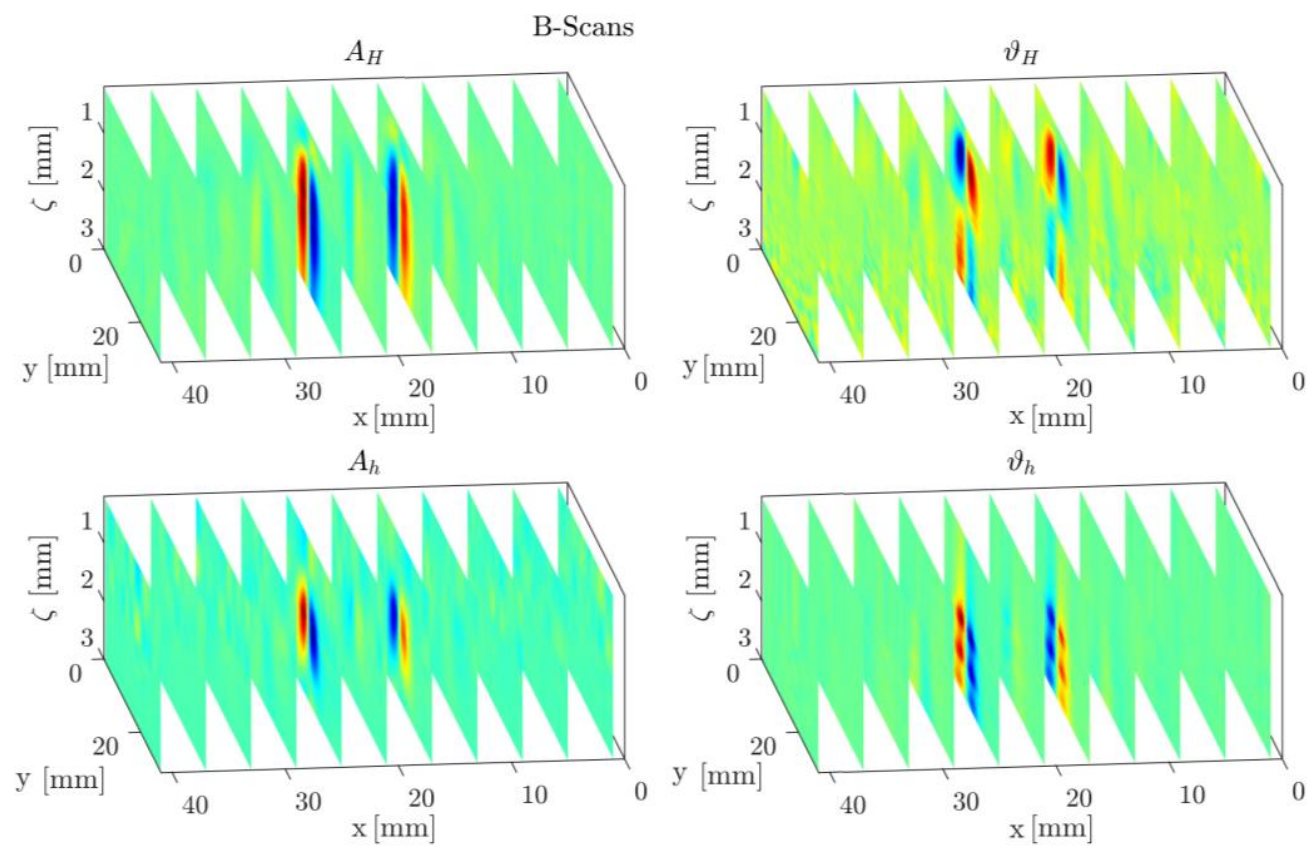

Fig. 6. B-scan $(y-\zeta)$ visualization for the defect $\mathrm{D}_{5}$ for all the four features. It can be noted that each feature produces different patterns 


\section{Experimental Results}

Once all the scans were acquired, the first analysis implemented was the visualization of the B-scan for all the features $\left(A_{H}, \vartheta_{H}, A_{h}, \vartheta_{h}\right)$ for the various defects and for each scan line. As example of such analysis, Figure 6 reports the $y-\zeta \mathrm{B}$-scans obtained for the defect $\mathrm{D}_{5}$ at various $x$ values for all the features. In all the cases, the detect was clearly visible even if amplitude features $A_{H}$ and $A_{h}$ produced images with higher contrast than phase features $\vartheta_{H}$ and $\vartheta_{h}$. It is also possible to see that the defect's pattern had a different shape for each feature, and this was verified for $x-\zeta \mathrm{B}$-scans and for all the defects as well. As example, Figure 7 depicts the best $y-\zeta$ B-scan images for the all the defects $D_{1}-D_{8}$ and for each feature. It was not straightforward to quantitatively compare the images quality and the effectiveness of the various features in terms of defect detection and characterization. On the other hand, by visualizing $x-y \mathrm{C}$-scans at different $\zeta$ values, strong similarities emerged between the images produced by all the features that made possible a quantitative comparison.

Recently, some of the authors [14,17,35], showed that C-scan images of $A_{H}$ and $\vartheta_{H}$ features are characterized by patterns closely resembling Hermite-Gaussian (HG) modes. The HG order depends on the relative orientation of sample surface, coil axis and the measured B component [36,37]. Precisely, for small defects, i.e. in the limit of validity of the Born approximation for the field scattered by the defect, the defect's image pattern is well approximated by the 2D convolution of the true geometric shape of the defect with a characteristic HG mode acting as Point Spread Function (PSF) [35]. It was also shown that for the present setup, the natural mode is a $H G_{(1,1)}$ mode of order $(1,1)$ characterized by a quadrupolar pattern that expands laterally as the frequency decreases.

Once the imaging procedure of Section III was applied, it was found that also $A_{h}$ and $\vartheta_{h}$ images are characterized by the same $H G_{(1,1)}$ pattern, as it is illustrated in Figure 8 for the same defect $\mathrm{D}_{5}$. Therefore, this
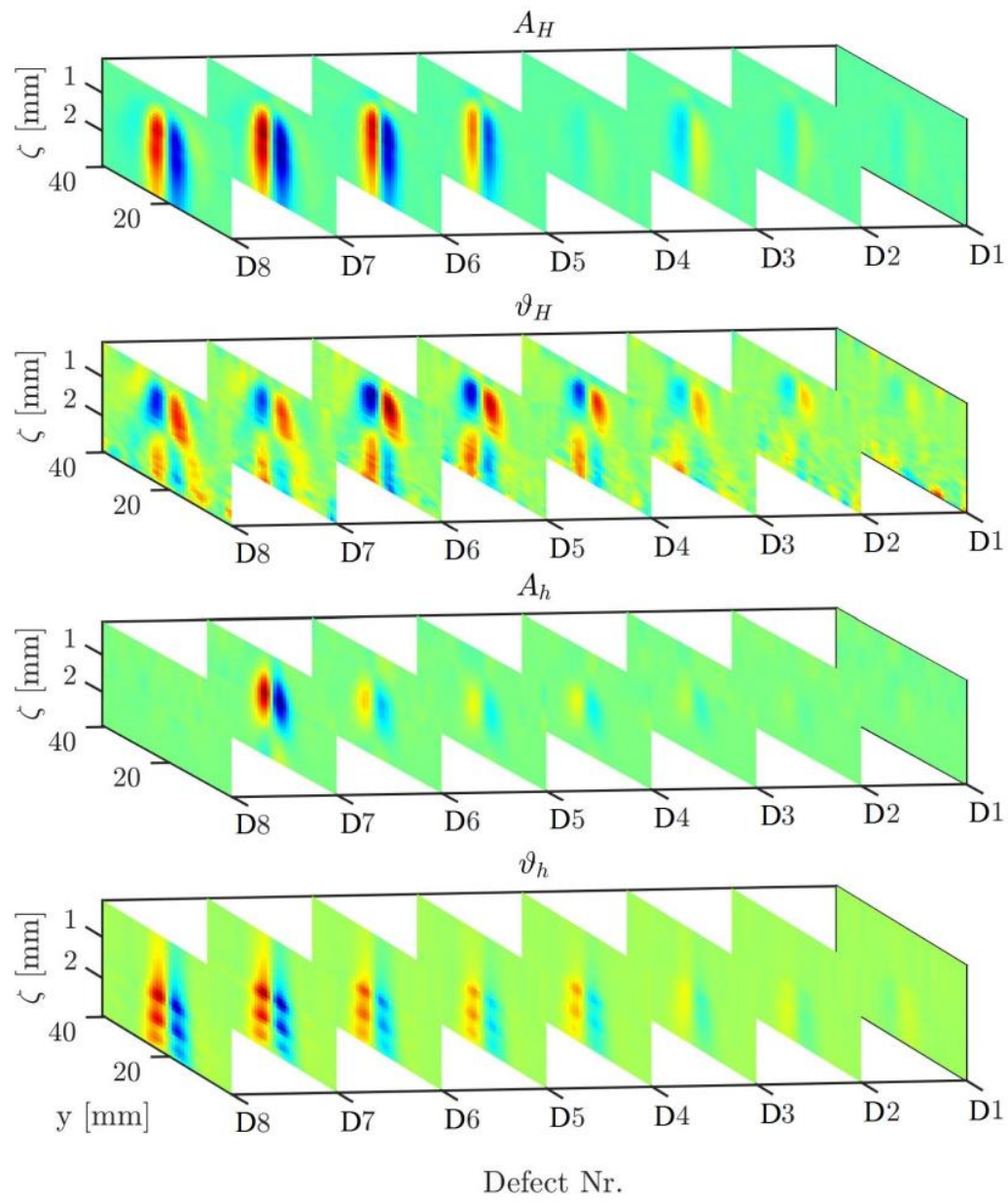

Fig. 7. Stack of the B-Scans $(y-\zeta)$ for the eight defects and for all the features. Each slice represents the B-Scan for the maximum SNR value for each defect and feature 

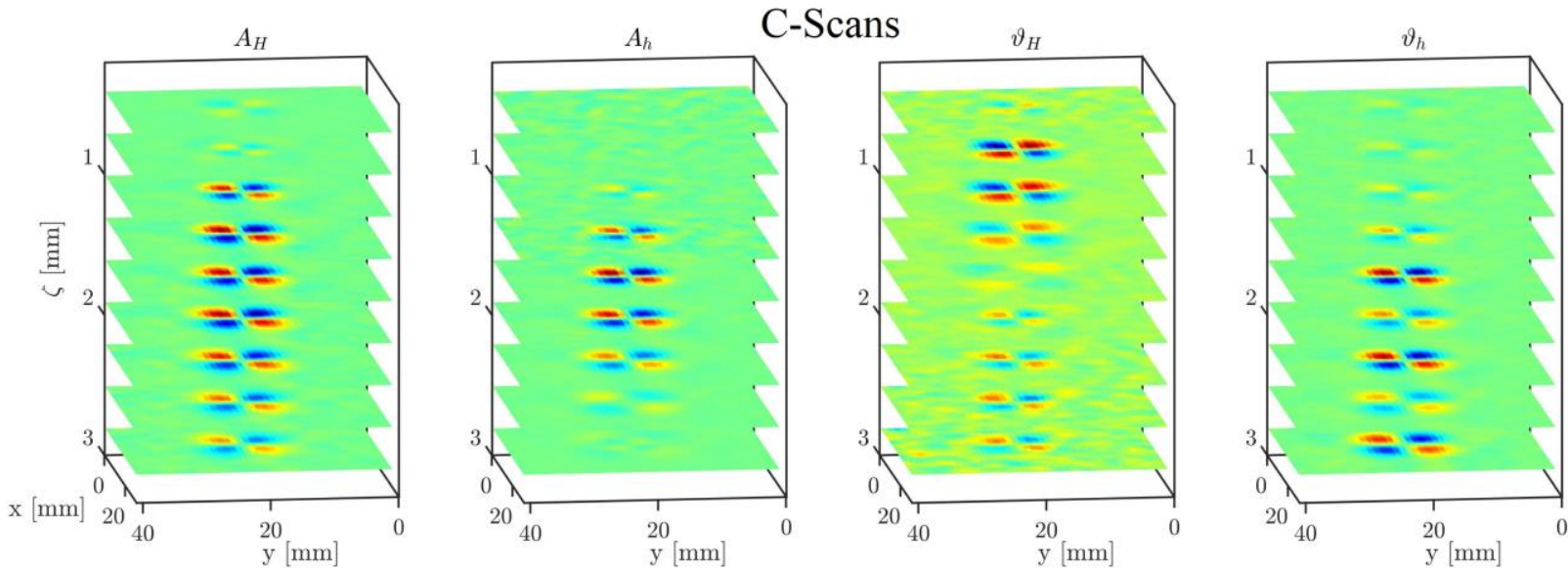

Fig. 8. $x-y$ visualization of the $\mathrm{C}$-scans measured for the defect $\mathrm{D}_{5}$. For all the features the defect pattern is very similar and well described by a Hermite-Gauss mode

fact was verified for all the defects, while extending the results reported in [14,17] and confirming that each sensor exhibits a characteristic HG pattern.

Figure 9 summarizes the characteristics of the various imaging procedures by showing the best $y-\zeta$ Bscan (Figure 9(a)) and $x-y$ C-scan images (Figure 9(b)) obtained for $\mathrm{D}_{5}$ for all the features. Except the sign and the noise level, the C-scan defect patterns are essentially the same while B-scan defect patterns are significantly different from each other. If the image analysis is limited to C-scans, the HG hypothesis can be extended to all the features and this is useful since allows analysing all the various images with the same processing algorithm and it makes easy to quantitatively compare them in terms of images' SNR, henceforth denoted as $I M_{S N R}$. The same image processing procedure and the same definition of $I M_{S N R}$ introduced in [14] were indeed exploited, both relying on the a-priori assumption of the $H G_{(1,1)}$ characteristic: for each defect/feature, a 2D bandpass filter is applied to each C-scan corresponding to a different $\zeta_{m}-$ th depth value. Then, for each depth $\zeta_{m}$, the $I M_{S N R}\left(\zeta_{m}\right)$ value is calculated as quantitative measure of the "image goodness".

Precisely, for each 2D image, the $I M_{S N R}$ value was defined as:

$$
I M_{S N R}=\frac{\max \left\{\left|I M_{\Gamma}\right|\right\}-\operatorname{mean}\left\{I M_{\bar{\Gamma}}\right\}}{\operatorname{std} I M_{\bar{\Gamma}}}=\frac{\mu_{\mathrm{IM}}}{\sigma_{\mathrm{IM}}}
$$

where $\Gamma$ and $\bar{\Gamma}$ are complementary parts of the image containing and not containing the defect respectively.

To quantitatively compare time- and frequency-domain imaging procedures, for each feature the resulting SNR values $I M_{S N R}\left(\zeta_{m}\right)$, were analysed as a function of $\zeta$. The resulting trends provides the means to

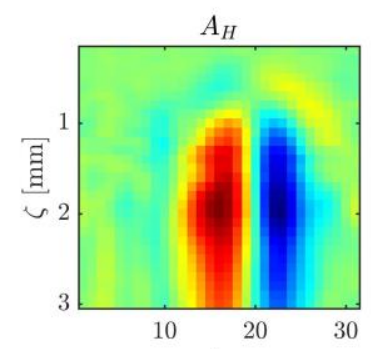

$A_{h}$

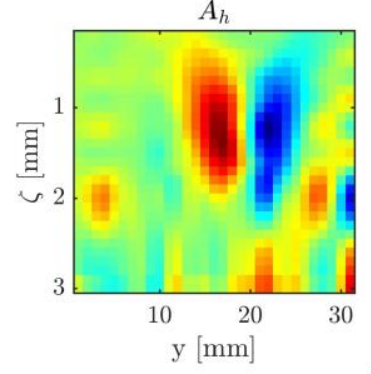

(a)

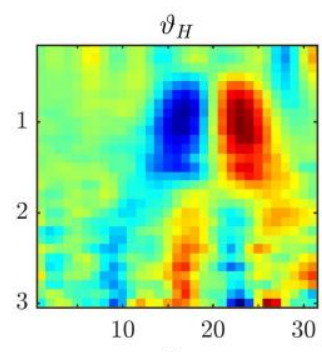

$\vartheta_{h}$

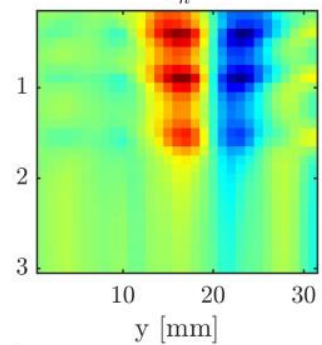

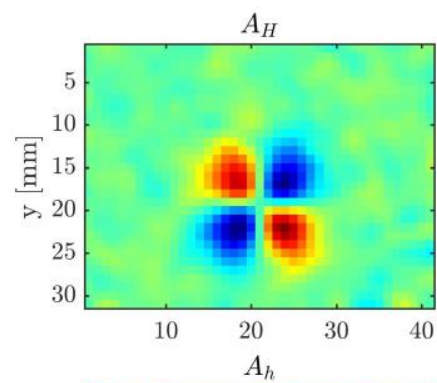

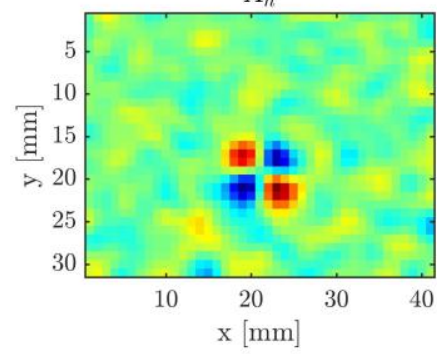

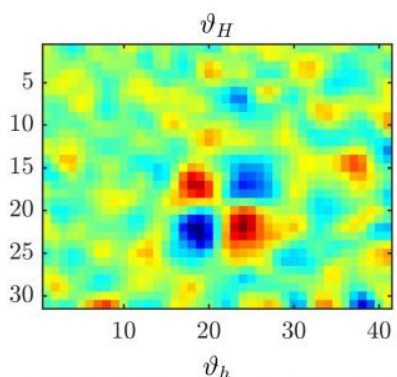

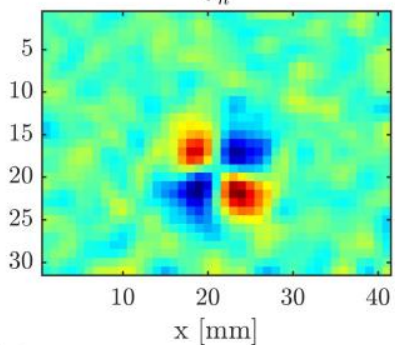

(b)

Fig. 9. Optimal images obtained for the defect $\mathrm{D}_{5}$ starting from the B- and C- scans reported in figures 6 and 8 . a) best B-scans and b) best C-scans corresponding to $I M_{S N R}\left(\zeta^{*}\right)$ The image reported are those exhibiting the highest images 


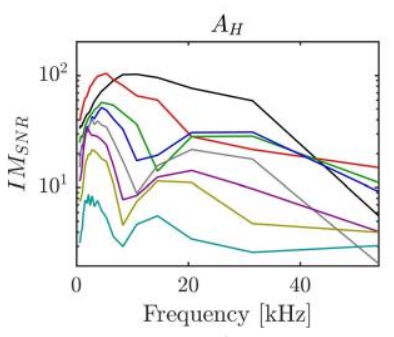

$A_{h}$
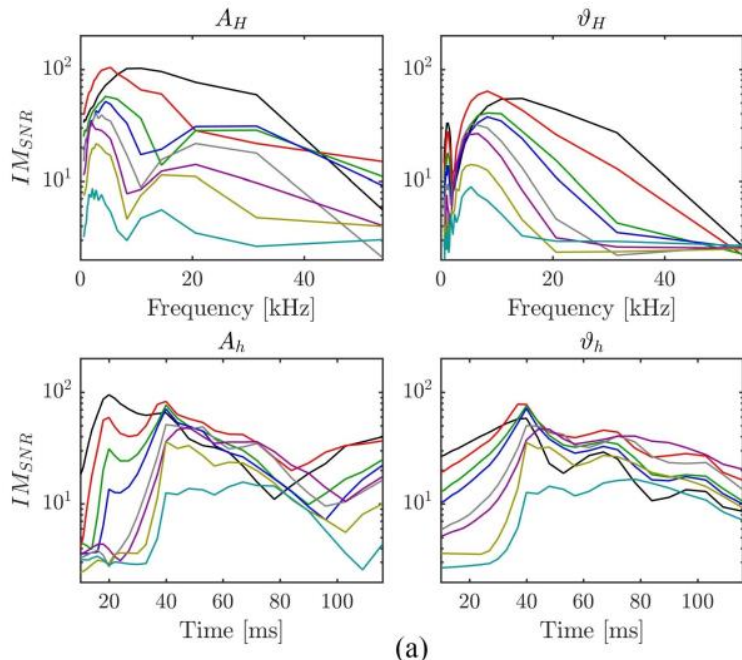

$\vartheta_{h}$

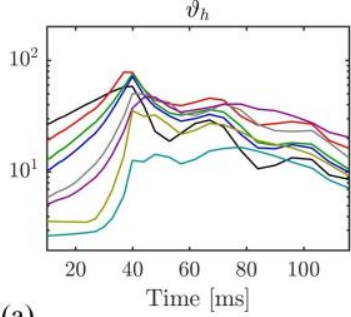

(a)
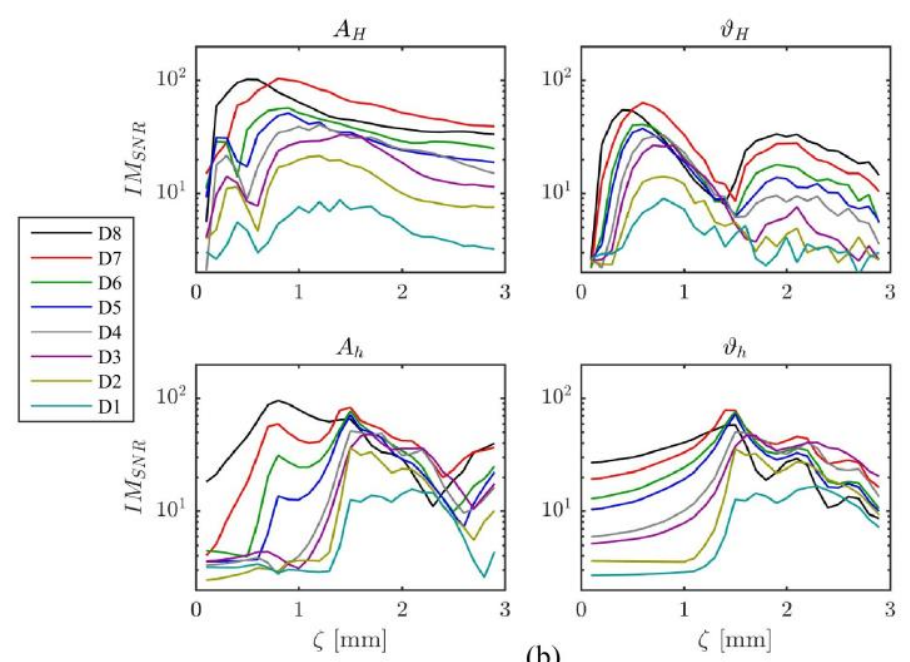

Fig. 10. $I M_{S N R}$ curves reconstructed for all the defects and for all the features as a function of the frequency and time (a) and as a function of the inspection depth $\zeta$ (b).

compare the defect detection capability of the various features and to quantitatively evaluate their defect characterization capability. For instance, the maximum $I M_{S N R}\left(\zeta^{*}\right)$ value achieved for a given defect and for a given feature was selected as the indicator of the "defect detection capability", since this parameter quantifies how better the defect is detected and visualized by the specific imaging procedure. The feature exhibiting the highest SNR peak value can be thus considered as the best one in terms of defect detection. Moreover, some characteristics of these trends such as the $\zeta^{*}$ value at which $I M_{S N R}$ is maximum, the riseand fall- slope of the $I M_{S N R}$ curves as well as their onset and offset values can be correlated with defect properties such as the depth. The analysis of the $I M_{S N R}$ curves for all the defects and for all the features allowed a first significant comparison between the effectiveness of the time-domain and the frequencydomain approaches, leading to quite interesting results.

In addition to this, following the analysis reported in [17], the imaging procedure was applied and the $I M_{S N R}$ curves calculated even in the case of random-varying LO value during scans. This allowed the comparison of the robustness of the various features with respect to LO variation. The analysis of $I M_{S N R}$ curves are reported in the next subsections

\section{VI.1. Fixed lift-offs}

For all the defects $\left(D_{1}-D_{8}\right)$, scans were acquired with a fixed $L O$ value of $0 \mathrm{~mm}$, corresponding to the minimum distance between the sensor case and the SUT. For all the features and for all the defects the $I M_{S N R}(\zeta)$ curves are plotted in Figure 10 while the maximum $I M_{S N R}\left(\zeta^{*}\right)$ values are reported in the bar plot of Figure 11

$\mathrm{A}_{h}$ and $\vartheta_{h}$ provide significantly higher $I M_{S N R}$ values than $A_{H}$ and $\vartheta_{H}$ for deepest defects $\left(\mathrm{D}_{1}-\mathrm{D}_{6}\right)$, revealing that time-domain imaging exhibits a better defect detection capability for the proposed analysis procedure in these cases. $A_{H}$ and $A_{h}$ give higher $I M_{S N R}$ values than the corresponding phase features $\vartheta_{H}$ and $\vartheta_{h}$ even though the latter exhibit more regular trends that could be better used to estimate some defect parameters such as the depth.

As depicted in Figure 10 left, if $I M_{S N R}$ curves for $\vartheta_{H}$ be plotted as a function of frequency (in dB scale), it will show an almost linear decay after the peak whose slope decreases monotonically as the depth increases. Once the $I M_{S N R}$ curves plotted as a function of the inspection depth $\zeta$, this characteristic is represented by a linear increasing onset depth $\zeta_{o n}$. Instead, $A_{h}$ and $\vartheta_{h}$ curves show respectively onset times and rising slopes that vary almost linearly with the defects' depth. A quantitative analysis of the correlation between these $I M_{S N R}$ curves' features and the defects' depth is beyond the scope of the present paper and it will be thoroughly elaborated in a subsequent work, however, by looking more carefully at Figure 10, it is possible to note that:

(i) the difference in the $I M_{S N R}$ peak values between amplitude and phase features decreases as the defect's depth increase and for $\mathrm{D}_{1}$ phase features are slightly better than amplitude ones; 


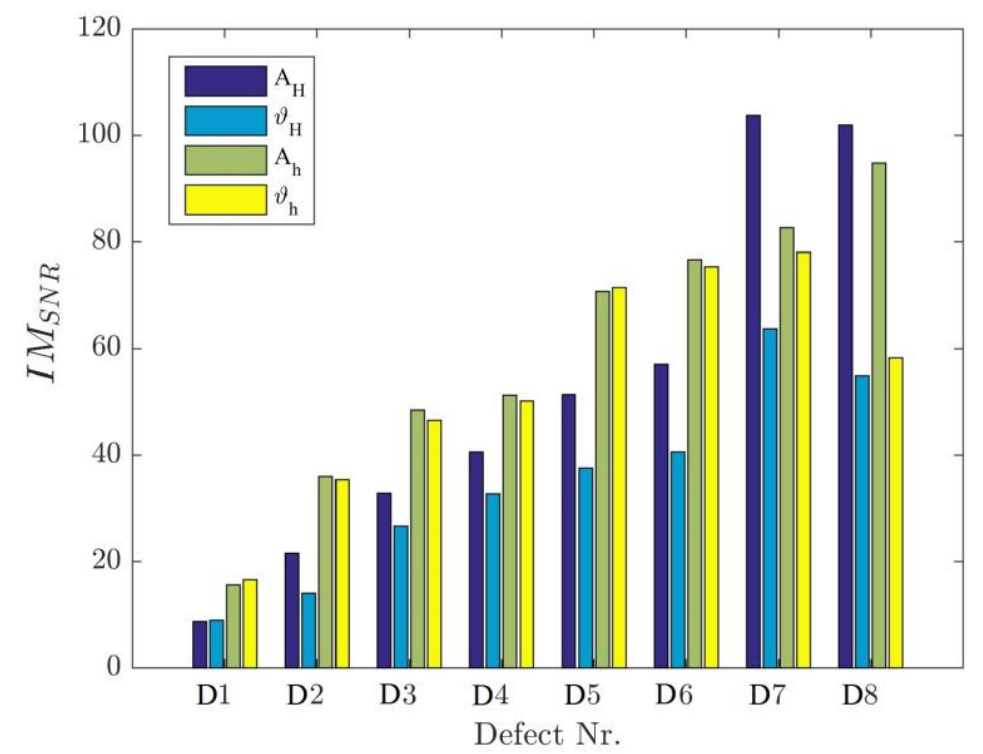

Fig. 11. Maximum SNR values for the four features and for all the defects

(ii) the difference in the $I M_{S N R}$ peak values between time and frequency features increases as the defect's depth increases;

(iii) the $I M_{S N R}$ peak value changes non-linearly with the defect depths reaching a plateau for the shallowest defect.

Furthermore, besides considering defect detection capability and depth estimation, it is also worth to analyse the spatial resolution of the imaging procedure and if and how defects' patterns change with the depth. Figure 12 reports the C-scan images corresponding to the maximum SNR value, i.e. $I M_{S N R}\left(\zeta^{*}\right)$ for all features of defects $D_{1}, D_{3}, D_{5}$ and $D_{7}$. The HG pattern shape is common to all defects even if it is larger for deeper defects, confirming a worsening of the EC spatial resolution as the inspection depth increases. This is expected due to the diffusion nature of eddy currents, but some strategies were proposed to increase spatial
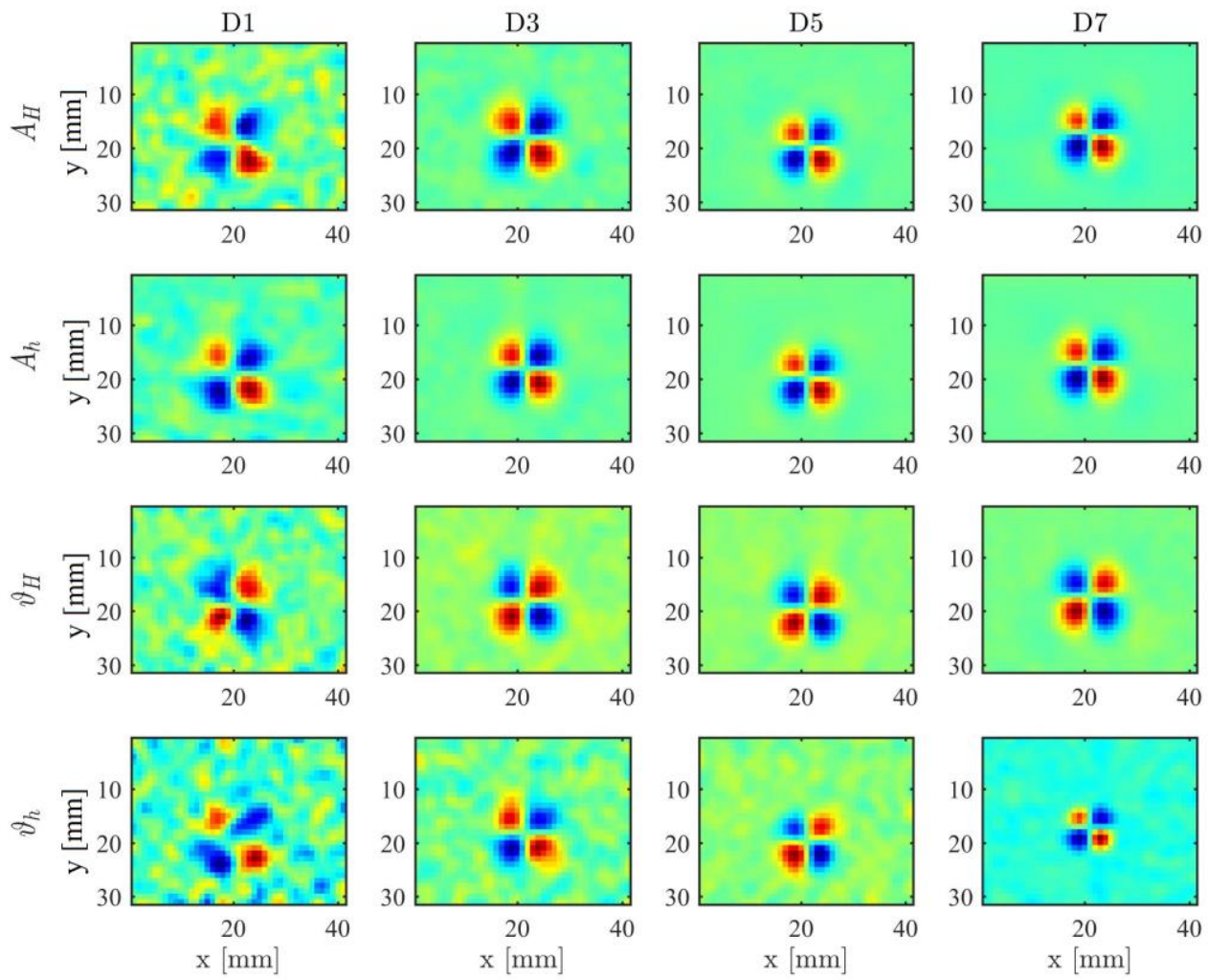

Fig. 12. C-scan images at maximum SNR values for the four features and for the defects $D_{1}, D_{3}, D_{5}$ and $D_{7}$ 
resolution by considering the Hermite-Gaussian hypothesis [35] or the use of the so-called Q-transform [38]. In perspective, the combination of both these approaches can significantly improve the $x-y$ and the $\zeta$ resolution. It would be also worth applying the virtual wave approach developed for thermography together with the proposed imaging procedures [39].
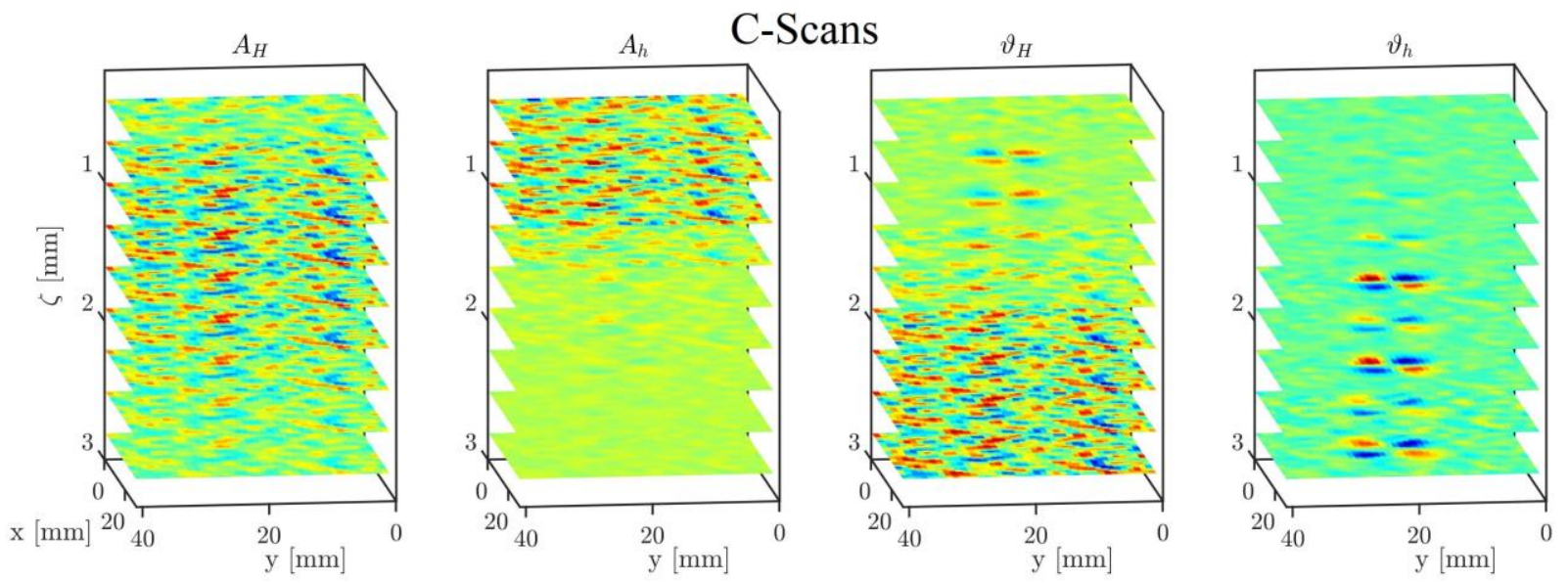

Fig. 13. $x-y$ visualization of the C-scans measured for the defect D5 in the case of random LO variation between 0 and $3 \mathrm{~mm}$

\section{VI.2. Random Lift-offs}

As stated earlier, we compared the time- and frequency-domain analysis also in the presence of random LO variations, following the same strategy used in [17]. For defects from $\mathrm{D}_{5}$ to $\mathrm{D}_{8}$, scans were collected at different $\mathrm{LO}$ values $\left(L O_{0}=0 \mathrm{~mm}, L O_{1}=1 \mathrm{~mm}, L O_{2}=2 \mathrm{~mm}, L O_{3}=3 \mathrm{~mm}\right)$ and then, for the imaging procedure, for each measurement point a LO value was selected randomly among the four. Therefore, starting from real experimental data, a scan affected by a very strong $\mathrm{LO}$ variation noise was simulated. For example, Figure 13 illustrates how the C-scans of the defect $\mathrm{D}_{5}$ are affected by the random LO. By comparing these images with those depicted in Figure 8, it can be seen that $A_{H}$ and $A_{h}$ images are strongly affected by LO so that at a first glance no defect pattern can be distinguished among the noise. On the other hand, $\vartheta_{H}$ and especially $\vartheta_{h}$ images are deteriorated by noise but still preserve enough SNR to detect the defects.

The robustness of $\vartheta_{H}$ feature against LO noise was already demonstrated in [17]. Figures 13 and 14 demonstrate that the proposed $\vartheta_{h}$ feature for ECT is not only robust against LO noise, but also it provides an even stronger robustness than phase imaging in frequency domain. Furthermore, by carefully analysing $\mathrm{A}_{h}$ images, it can be noted that for some specific depth/time values (time interval index equal to 8), the defect pattern can be clearly distinguished with SNR values comparable with the ones obtained by the phase features.

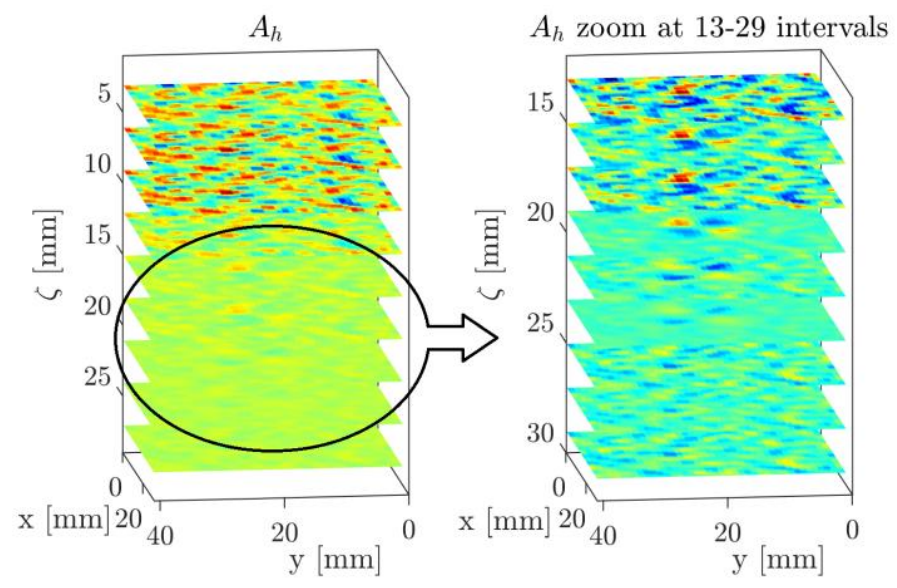

Fig. 14. C-scan feature for TA of defect D5 (left), and zoom view of the defect area (right) 
This phenomenon is highlighted in Figure 14 that visualizes a zoom of the $A_{h} C$-scan in a specific inspection depth (i.e. time) interval. For particular $\zeta$ values, the $A_{h}$ images are significantly less affected by LO noise. We hypothesize that these values correspond to the time intervals in which LOI points fall in the reconstructed PEC response after PuC [40-41].
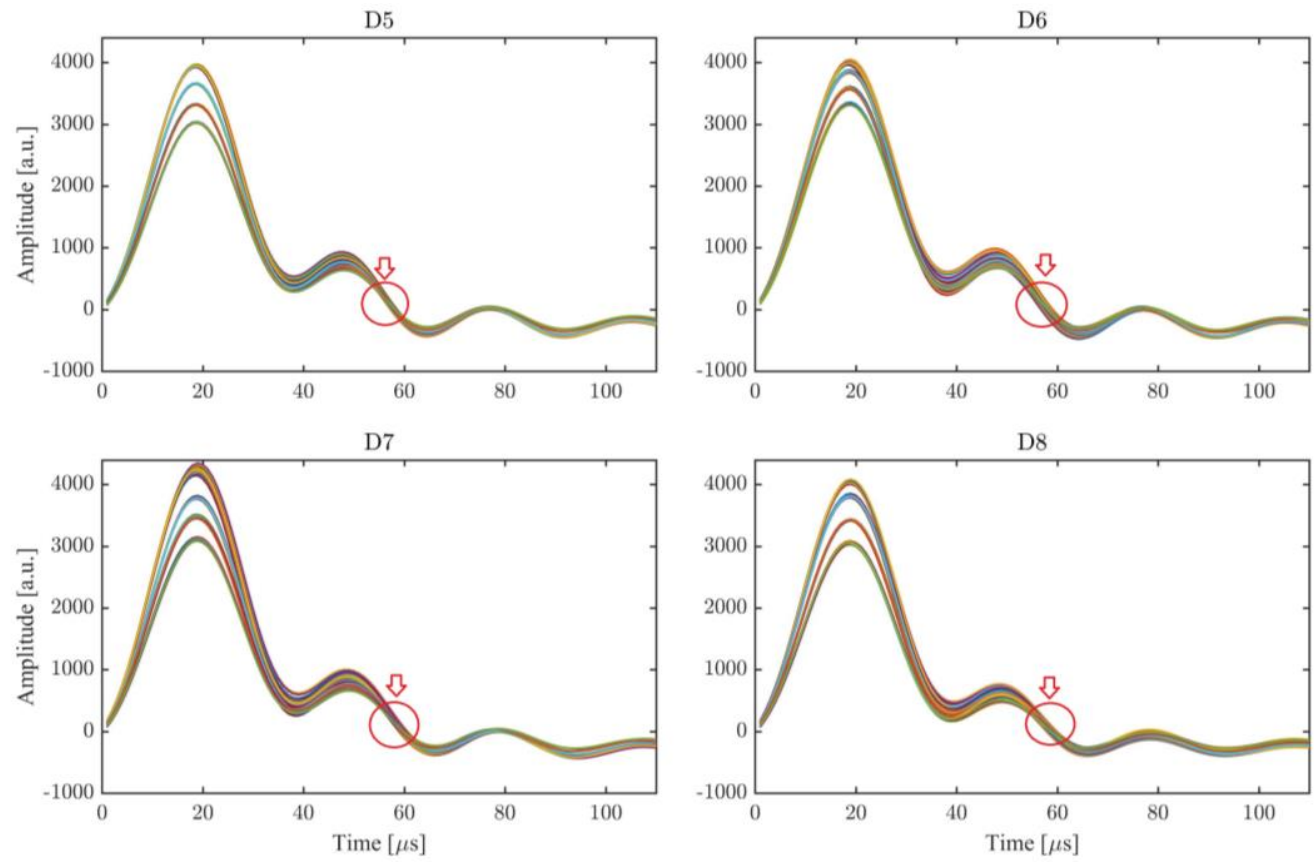

Fig. 15. Impulse response $h(t)$ of defects $\mathrm{D}_{5}$ to $\mathrm{D}_{8}$ for $\mathrm{LO}$ values from $0 \mathrm{~mm}$ to $3 \mathrm{~mm}$. Red circles indicate the LOI points

To confirm this hypothesis, Figure 15 reports various $h_{P u C}\left(t, x_{k}, y_{l}\right)$ signals (see Eq. 6) obtained at several scan points and for each LO value. The effect of LO variation is evidenced by the four different amplitude levels of the signals. However, for some time intervals, as the highlighted in the plot by the red solid circles, all the signals cross almost at the same value, which is assumed to be the LOI point. Other LOI points or regions can be also identified at different time values. The $A_{h}$ images corresponding to time intervals $\vec{t}_{k}=t^{*}\left(\vec{\zeta}_{k}\right)$ in which a LOI point falls, exhibit robustness against LO noise as well.

These results to our knowledge represent the first experimental evidence of LOI point not found by PEC or a single-tone excitation but instead retrieved from coded excitation and after the application of a remarkable filter, i.e. PuC. Furthermore, this fact confirms that PuC-ECT can replace the standard PEC without losing the attractive features of the technique while providing higher SNR values. This observation is also in line with the LOI phenomenon general behaviour in ECT reported by other authors $[42,43]$ since it may represent the diffusive nature of the eddy currents in general.

To evaluate the robustness of the time- and frequency-domain imaging features against LO variations, several scans affected by random LO were simulated by hundred times repetition of the random process and plotting the SNR curves for all the repetitions versus the inspection depth $\zeta$. The simulations were carried out by starting from experimental data collected for each $x-y$ point at various LO values. By following the same strategy described in Section IV of [17], hundred random cases were made through a repetition loop.

In Figure 16 all the $I M_{S N R}$ curves retrieved from the hundred simulated scans with random LO are plotted in grey, their mean value is solid black line and $I M_{S N R}$ curves attained at various fixed LO values are solid colour lines.

It is clearly evidenced that:

(i) the $\mathrm{A}_{H}$ feature is highly affected by LO noise and for the tough working conditions simulated, it is completely unable to detect even the shallowest defect, as showed in [17] for MF-ECT data;

(ii) the $\vartheta_{H}$ feature instead is very robust against LO and the $I M_{S N R}$ curves remain quite regular and depth-dependent also in the extreme simulated scenario, see [17];

(iii) the $\vartheta_{h}$ feature also is very robust against LO and the $I M_{S N R}$ curves achieve higher value than those obtained for $\vartheta_{H}$ in the case of deepest defects; 
(iv) the $A_{h}$ feature also is in general not robust against LO except for the inspection depths corresponding to LOI points.
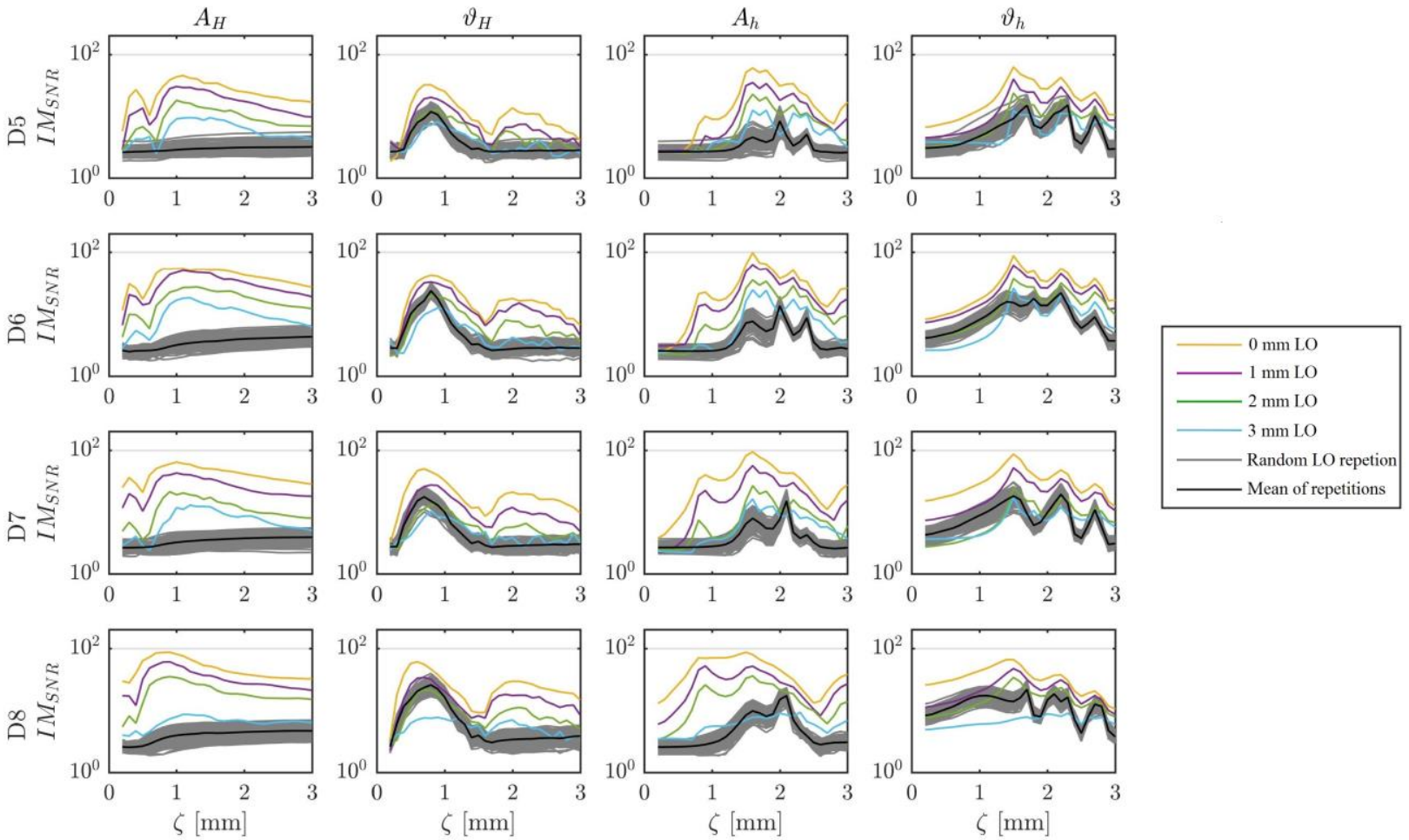

Fig. 16. $I M_{S N R}$ curves obtained in presence of random LO noise for all features and for $\mathrm{D}_{5}-\mathrm{D}_{8}$ as a function of the inspection depth $\zeta . I M_{S N R}$ curves retrieved from hundred simulated in grey; their mean value is plotted with the black line; $I M_{S N R}$ curves attained at various fixed LO values are coloured.

This latter point implies that for the $\mathrm{A}_{h}$ feature in presence of LO noise, the $\zeta^{*}$ value at which $I M_{S N R}$ reaches its maximum cannot be used to estimate the defect depth since it is forced by LOI. On the other hand, the $\vartheta_{h}$ feature on average seems to be the better one in terms of detection capability and LO robustness.

\section{Conclusion and future work}

In this work, a comparison between PuC-ECT imaging procedures relying on both time- and frequencydomain analysis was performed based on experimental data collected on a benchmark sample. A sweptfrequency chirp excitation signal was used, and the time analysis was implemented on the signals retrieved after the application of the pulse-compression procedure and and of the Hilbert transform. Experimental data were collected at different LO values and were used to evaluate the features in fixed and simulated random LO cases. The results showed that the adopted scheme allows the contextual exploitation of the advantages of both PEC and MF-ECT methods. Higher amount of energy delivered by the chirp excitation signal with respect to pulsed excitation assures high SNR values and hence an improvement of defect detection capability. The advantages of PEC approach with respect to MF-ECT (high sensitivity to deep defects, LO noise robustness and LOI point) are retrieved using PuC as well. For fixed LO values, time and frequency domain features give similar results in terms of defect detection, although time-domain imaging features exhibit a quite better SNR than the frequency-domain ones for deeper defects, i.e. $\mathrm{D}_{6}$ to $\mathrm{D}_{1}$.

For random variable LO values, phase feature of both time- and frequency-domain imaging are very robust against the LO noise, while amplitude feature especially in frequency domain is strongly degraded as it was shown through comparison of the $\mathrm{IM}_{\mathrm{SNR}}$ curves for defects $\mathrm{D}_{5}$ to $\mathrm{D}_{8}$. The frequency amplitude was not able to detect even the shallowest defect $\mathrm{D}_{8}$ in presence of the random LO noise while other features were able to detect the defects, even though with different $\mathrm{IM}_{\mathrm{SNR}}$ values. Despite this general behaviour, timeamplitude feature can exploit the presence of LOI points. The images obtained for the time intervals in which the LOI points fall, are very robust to LO noise providing SNR value comparable or higher than the phase 
features. In general, the proposed time-phase feature seems to be a good and reliable tool for the improvement in defect detection capability of PuC-ECT.

In the future, the PuC procedures will be further optimized, and spatial and depth resolution of imaging procedures will be increased. By using Hermite-Gauss deconvolution [35] the shape of the defect on the $x-$ $y$ plane can be estimated while the use of the Q-transform together with the virtual wave approach can lead to a significant improvement of the image resolution along the $\zeta$ axis [38-39]. In addition to the improvement in post-processing algorithms, some hardware and software improvements will be beneficial to achieve better detectability, depth and lateral resolution. For example, the use of array of sensors can reduce acquisition time while further increasing the defect detection capability and the lift-off robustness. Machine learning techniques can also help on fruitfully fuse and integrate the information provided by the time- and frequency-domain analysis.

It will be also of interest to explore the possibility of combining various images to improve the ECT performances in terms of both defect detection and classification.

\section{Acknowledgement}

This research work has been partially supported from the European Union's Horizon 2020 research and innovation programme under the Marie Skłodowska-Curie grant agreement No 722134 - NDTonAIR.

The authors acknowledge Prof. Pietro Burrascano for the useful suggestion about the optimal window function to use in the ECT pulse-compression algorithm.

\section{Bibliography}

[1] Lebrun B, Jayet Y, Baboux J.C, "Pulsed eddy current signal analysis: application to the experimental detection and characterization of deep flaws in highly conductive materials" NDT \& E International, 30 (3), 163-170 (1997).

[2] Glorieux C, Moulder J, Basart J, \& Thoen $\mathbf{J}$ "The determination of electrical conductivity profiles using neural network inversion of multi-frequency eddy-current data”, Journal of Physics D: Applied Physics, 32 (5), 616. (1999).

[3] Chady T, Enokizono $M$ and Sikora $\mathrm{R}$ "Crack detection and recognition using an eddy current differential probe", IEEE Transactions on Magnetics, 35 (3), 1849-1852 (1999).

[4] Sophian A, Tian G.Y, Taylor D, and Rudlin J,"Design of a pulsed eddy current sensor for detection of defects in aircraft lap-joints", Sensors and Actuators A: Physical, 101 (1), 92-98 (2002).

[5] Yin W, Dickinson S.J and Peyton A.J "A multi-frequency impedance analysing instrument for eddy current testing", Meas. Sci. Technol, 17, 393-402, (2006)

[6] Le Diraison Y, Joubert P.Y, and Placko D "Characterization of subsurface defects in aeronautical riveted lap-joints using multi-frequency eddy current imaging." NDT \& E International, 42 (2), pp. 133-140 (2009).

[7] Yang G, Tamburrino A, Udpa L, Udpa S.S, Zeng Z, Deng Y, and Que P "Pulsed eddy-current based giant magnetoresistive system for the inspection of aircraft structures", IEEE Transactions on Magnetics, 46 (3), pp.910-917 (2010).

[8] Bernieri A, Ferrigno L, Laracca M, \& Molinara M "Crack shape reconstruction in eddy current testing using machine learning systems for regression", IEEE Transactions on Instrumentation and Measurement, 57 (9), 1958-1968, (2008).

[9] Hughes R, Fan Y, and Dixon S "Near electrical resonance signal enhancement (NERSE) in eddycurrent crack detection." NDT \& E International, 66, 82-89, (2014).

[10] Chady T, \& Enokizono M, "Multi-frequency exciting and spectrogram-based ECT method", Journal of Magnetism and Magnetic Materials, 215, 700-703, (2000).

[11] Yin W and Peyton A.J "Thickness measurement of non-magnetic plates using multi-frequency eddy current sensors", Ndt \& E International, 40 (1), 43-48, (2007).

[12] Bernieri A, Betta G, Ferrigno L, Laracca M "Crack Depth Estimation by Using a Multi-Frequency ECT Method," IEEE Transactions on Instrumentation and Measurement, 62 (3), 544-552, (2013)

[13] Joubert P.Y, Vourc'h E, \& Thomas V "Experimental validation of an eddy current probe dedicated to the multi-frequency imaging of bore holes", Sensors and Actuators A: Physical, 185, 132-138, (2012). 
[14] Betta G, Ferrigno L, Laracca M, Burrascano P, Ricci M, \& Silipigni G “An experimental comparison of multi-frequency and chirp excitations for eddy current testing on thin defects", Measurement, 63, 207-220, (2015).

[15] Hoshikawa H, Koyama K, \& Karasawa H " A new ECT surface probe without lift-off noise and with phase information on flaw depth”, AIP Conference Proceedings , 557 (1), 969-976, (2001).

[16] Ribeiro A.L, Ramos H.G, \& Arez J.C "Liftoff insensitive thickness measurement of aluminum plates using harmonic eddy current excitation and a GMR sensor", Measurement, 45(9), 2246-2253, (2012).

[17] Ricci M, Silipigni G, Ferrigno L, Laracca M, Adewale I.D, \& Tian G.Y "Evaluation of the lift-off robustness of eddy current imaging techniques", NDT \& E International, 85, 43-52, (2017).

[18] Sophian A, Tian G.Y, Taylor D, Rudlin J, "A feature extraction technique based on principal component analysis for pulsed Eddy current NDT", NDT \& E International, 36 (1), 37-41, (2003).

[19] He Y, Luo F, Pan M, Weng F, Hu X, Gao J, \& Liu B "Pulsed eddy current technique for defect detection in aircraft riveted structures", NDT \& e International, 43 (2), 176-181, (2010).

[20] Tian G.Y, \& Sophian A "Reduction of lift-off effects for pulsed eddy current NDT", NDT \& E International, 38(4), 319-324, (2005).

[21] Shu L, Songling H, Wei Z, \& Peng Y "Improved immunity to lift-off effect in pulsed eddy current testing with two-stage differential probes", Russian journal of nondestructive testing, 44 (2), 138-144, (2008).

[22] Shin Y.K, Choi D.M, Kim Y.J, \& Lee S.S "Signal characteristics of differential-pulsed eddy current sensors in the evaluation of plate thickness", NDT \& E International, 42 (3), 215-221, (2009).

[23] Yang B, Li B, \& Wang Y "Reduction of lift-off effect for pulsed eddy current NDT based on sensor design and frequency spectrum analysis", Nondestructive Testing and Evaluation, 25 (1), 77-89, (2010).

[24] Safizadeh M.S, Lepine B.A, Forsyth D.S, \& Fahr A "Time-frequency analysis of pulsed eddy current signals", Journal of nondestructive evaluation, 20 (2), 73-86, (2001).

[25] Morozov M, Tian G.Y, \& Edgar D "Comparison of PEC and SFEC NDE techniques", Nondestructive Testing and Evaluation, 24 (1-2), 153-164 (2009).

[26] Burrascano P, Carpentieri M, Pirani A, \& Ricci M "Galois sequences in the non-destructive evaluation of metallic materials", Measurement Science and Technology, 17 (11), 2973, (2006).

[27] Udpa L, \& Lord W "Diffusion, waves, phase and eddy current imaging". In Review of Progress in Quantitative Nondestructive Evaluation (pp. 499-506). Springer, Boston, MA, (1985).

[28] Stratton, J.A "Electromagnetic theory", John Wiley \& Sons, (2007).

[29] Bossavit A "Computational electromagnetism: variational formulations, complementarity, edge elements" Academic Press, (1998).

[30] Knoepfel, H.E "Magnetic fields: a comprehensive theoretical treatise for practical use" John Wiley \& Sons, (2008).

[31] Tabatabaei N, and Mandelis A "Thermal coherence tomography using match filter binary phase coded diffusion waves", Physical review letters, 107 (16), 165901, (2011).

[32] Arora V, Siddiqui J.A, Mulaveesala R, Muniyappa A. "Hilbert transform-based pulse compression approach to infrared thermal wave imaging for sub-surface defect detection in steel material." InsightNon-Destructive Testing and Condition Monitoring, 56 (10), 550-552, (2014).

[33] Pollakowski M, and Ermert H. "Chirp signal matching and signal power optimization in pulse-echo mode ultrasonic nondestructive testing." IEEE transactions on ultrasonics, ferroelectrics, and frequency control , 41 (5), 655-659, (1994).

[34] Burrascano P, Laureti S, Senni L, Ricci M. "Pulse Compression in Nondestructive Testing Applications: Reduction of Near Sidelobes Exploiting Reactance Transformation" IEEE Transactions on Circuits and Systems I: Regular Papers 99,1-11, (2018).

[35] Betta G, Ferrigno L, Laracca M, Ramos H.G, Ricci M, Ribeiro A.L, Silipigni G. "Fast 2D crack profile reconstruction by image processing for Eddy-Current Testing," Proceeding of IEEE International Workshop on Metrology for Aerospace, Benevento, Italy, pp. 356-360, 4-5 June 2015

[36] Kogelnik H, and Li T. "Laser beams and resonators." Applied Optics 5 (10), 1550-1567, (1966).

[37] Hermite-Gaussian beams are similar to Gaussian ones, except that the electric field amplitude is modulated in the transverse direction according to: $E(x, y)=A H_{m}\left(\frac{\sqrt{2}}{w} x\right) H_{n}\left(\frac{\sqrt{2}}{w} y\right) \exp \left(-\frac{x^{2}+y^{2}}{w^{2}}\right)$ where w is the spot size at the position $\mathrm{z}$, and the $H_{k}(u)$ are Hermite polynomials [27]. For our purpose, it is sufficient to know that these are well-known polynomials of order $\mathrm{m}$, which are the solution to a 
particular differential equation. For example, these functions turn up in the solution of the harmonic oscillator problem in quantum mechanics. The first few Hermite polynomials are: $\mathrm{H} 0(\mathrm{u})=1$, $\mathrm{H} 1(\mathrm{i})=2 \mathrm{u}, \mathrm{H} 2(\mathrm{u})=4 \mathrm{u} 2-2, \mathrm{H} 3(\mathrm{u})=8 \mathrm{u} 3-12 \mathrm{u}$.

[38] Tamburrino A, Fresa R, Udpa S.S, \& Tian Y. "Three-dimensional defect localization from time-offlight/eddy current testing data", IEEE transactions on magnetics, 40 (2), 1148-1151, (2004).

[39] Burgholzer P, Thor M, Gruber J, \& Mayr G. "Three-dimensional thermographic imaging using a virtual wave concept", Journal of Applied Physics, 121 (10), 105102, (2017).

[40] Tian G.Y, Li Y, \& Mandache C. "Study of lift-off invariance for pulsed eddy-current signals", IEEE transactions on magnetics, 45 (1), 184-191, (2009).

[41] Fan M, Cao B, Tian G, Ye, B, \& Li W. "Thickness measurement using liftoff point of intersection in pulsed eddy current responses for elimination of liftoff effect", Sensors and Actuators A: Physical, 251, 66-74, (2016).

[42] Fan M, Cao B, Imam Sunny A, Li W, Tian G.Y, and Ye B. "Pulsed eddy current thickness measurement using phase features immune to liftoff effect". NDT\&E International, 2017, 86(3): 123131.

[43] Mandache C, and Lefebvre J.H.V. "Transient and harmonic eddy currents: lift-off point of intersection”, NDT\&E International. 39(1) (2006) 57-60. 